\title{
Adsorbate-Limited Conductivity of Graphene
}

\author{
John P. Robinson, Henning Schomerus, László Oroszlány, and Vladimir I. Fal'ko \\ Department of Physics, Lancaster University, Lancaster, LA1 4YB, United Kingdom
}

(Received 18 August 2008; published 5 November 2008)

\begin{abstract}
We present a theory of electronic transport in graphene in the presence of randomly placed adsorbates. Our analysis predicts a marked asymmetry of the conductivity about the Dirac point, as well as a negative weak-localization magnetoresistivity. In the region of strong scattering, renormalization group corrections drive the system further towards insulating behavior. These results explain key features of recent experiments, and are validated by numerical transport computations.
\end{abstract}

DOI: 10.1103/PhysRevLett.101.196803

PACS numbers: 73.63. $-\mathrm{b}, 72.10 . \mathrm{Bg}, 81.05 . \mathrm{Uw}$

Graphene (the two-dimensional allotrope of carbon [1]) offers a chemically stable platform to host various chemical adsorbates [2-7]. Their presence strongly affects electronic transport, making graphene-based devices suitable for chemical sensoring. A key observation in experiments on chemically functionalized samples is a marked asymmetry of the conductivity as a function of a back gate voltage, which is used to steer the system across the charge neutrality point (the Dirac point, which separates the valence band from the conduction band). The conductivity becomes symmetric only when the sample is annealed by a strong bias current which dislodges the adsorbates.

In this Letter we provide a theory, supported by numerical simulations, that explains this experimental feature for covalently bonded (chemisorbed) adsorbates [8-10]. Our model is based on the tight-binding description of electrons in graphene [11]. Chemisorbed molecules are incorporated into this description as laterally attached additional sites, where the on-site and coupling energies are extracted from the band structure of a graphene sheet with regularly placed adsorbates. Each type of adsorbate introduces a characteristic local energy-dependent scattering potential in the graphene, and suppresses the conductivity on one side of the Dirac point, with only a weak effect on the other. For example, adsorbed $\mathrm{H}^{+}$yields almost insulating behavior in $n$-type graphene, while in $p$-type structures the conductivity is close to that of clean material. For $\mathrm{OH}^{-}$ the role of the bands is reversed. When combined with scattering from a random Coulomb potential $[12,13]$, the resulting conductivity traces (shown in Fig. 1) are consistent with the findings in experiments $[5,6]$. For a small adsorbate concentration, these conclusions can be drawn from kinetic theory. For larger concentrations, we implement a recently proposed renormalization group (RG) analysis $[14,15]$ to account for systematic quantum corrections to the conductivity resulting from multiadsorbate scattering. Our predictions are in good quantitative agreement with the results of the numerical transport computations.

In a graphene sheet, the carbon atoms are held together via $s p^{2}$-hybridized covalent bonds, while the electronic transport takes place by hopping along $\pi$ orbitals which can participate in covalent bonding with adsorbates. The electrons in the $\pi$ band of graphene with additional adsorbed atoms can be described using a tight-binding Hamiltonian

$$
\begin{aligned}
\mathcal{H} & =-\gamma \sum_{\langle l, m\rangle} c_{l}^{\dagger} c_{m}+\sum_{n} \mathcal{H}_{n}, \\
\mathcal{H}_{n} & =\varepsilon_{i} d_{n}^{\dagger} d_{n}+\gamma_{i}\left(c_{\alpha_{n}}^{\dagger} d_{n}+c_{\alpha_{n}} d_{n}^{\dagger}\right) .
\end{aligned}
$$
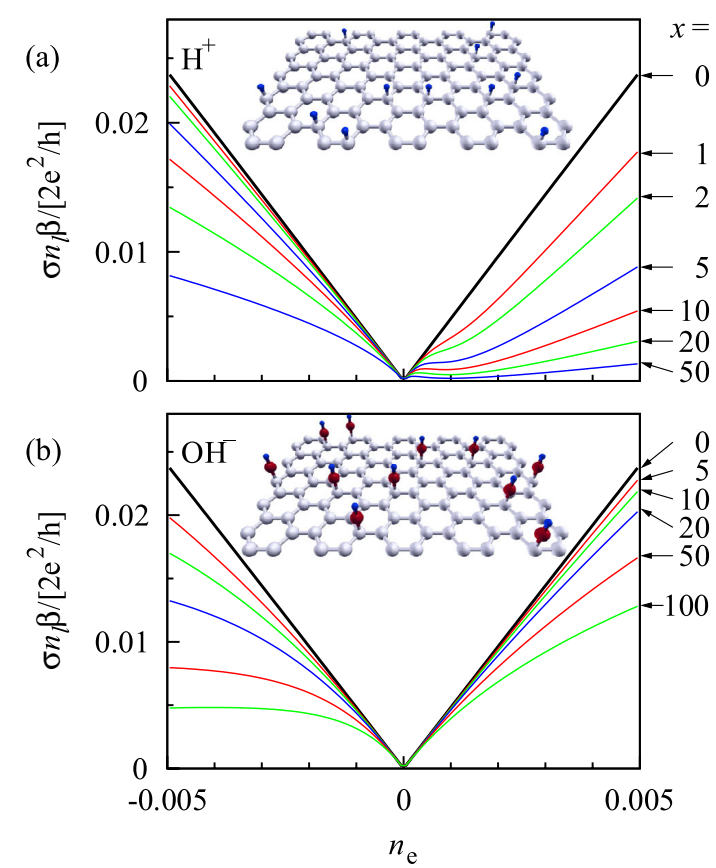

FIG. 1 (color online). Conductivity of graphene in the presence of adsorbates (concentration $n_{i}$ ) and Coulomb impurities (concentration $n_{l}$ ) as a function of charge-carrier concentration $n_{e}$ (carrier density $n_{e} / A_{c}$ with $A_{c}=3 \sqrt{3} a^{2} / 4$ the area per carbon atom), for various ratios $n_{i} / n_{l}=(\beta / 2 \pi) x$ where the dimensionless parameter $\beta \simeq 1$ characterizes the scattering strength of the Coulomb impurities $[12,13,25]$. (a) $\mathrm{H}^{+}$, (b) $\mathrm{OH}^{-}$, with parameters from first-principle computations (the insets illustrate sample segments). The results are based on Eq. (7). 
The first term of Eq. (1a) corresponds to the Hamiltonian of clean graphene, where $c_{l}$ are annihilation operators on sites of the honeycomb lattice, and the nearest-neighbor coupling strength $\gamma=2 \hbar v_{\mathrm{D}} / 3 a$ (with bond length $a=$ $1.42 \AA$ ) determines the Fermi velocity $v_{\mathrm{D}}$ at the Dirac point. Each adsorbed molecule contributes a term of the form (1b), where $d_{n}$ is the annihilation operator on the adsorbate site and $\alpha_{n}$ is the host position on the honeycomb lattice. The adsorbate density is parameterized by $n_{i} / A_{c}$, where $n_{i}$ is the adsorbate-to-carbon ratio, and $A_{c}=$ $3 \sqrt{3} a^{2} / 4$ is the area per carbon atom in graphene.

The model Hamiltonian (1) can be justified by firstprinciple calculations. Here we consider the adsorbates $\mathrm{H}^{+}$and $\mathrm{OH}^{-}$, chosen because of the presence of ambient water in many experiments, and which also can be selectively driven towards the graphene via electric fields [3]. Using density-functional theory (DFT) [16], we find that $\mathrm{H}^{+}$is described by $\varepsilon_{i}=0.66 \gamma, \gamma_{i}=2.2 \gamma$, while for $\mathrm{OH}^{-}$ $\varepsilon_{i}=-2.9 \gamma, \gamma_{i}=2.3 \gamma$. These energies are comparable to the graphene hopping energy $\gamma=2.6 \mathrm{eV}$, which necessitates the nonperturbative approaches employed in this Letter. Our DFT calculations also confirm that the adsorbates form covalent bonds to well-defined host sites. In the fully relaxed configuration, both adsorbates are aligned in the vertical direction of the graphene sheet (for illustration, see insets of Fig. 1). Other energy scales (such as shifts of the graphene on-site energies and next-to-nearest neighbor couplings) are small and can be safely neglected $[17,18]$.

The main building block of our analytical considerations is the derivation of the scattering amplitude due to individual adsorbates, which enters the collision term of kinetic theory and also features as input into the renormalization group analysis that captures quantum corrections due to multiple scattering.

In the first step we self-consistently eliminate the adsorbate sites from the Hamiltonian (1) via a decimation procedure. The electron wave function can be written as $|\Psi\rangle=\sum_{l} \psi_{l}|l\rangle+\sum_{n} \phi_{n}|n\rangle_{\mathrm{ad}} \equiv|\psi\rangle \oplus|\phi\rangle$, where the amplitudes $\psi_{l}$ and states $|l\rangle$ refer to the carbon sites, while $\phi_{n}$ and $|n\rangle_{\mathrm{ad}}$ refer to the adsorbate sites. We now project the Schrödinger equation onto an adsorbate site, $\left\langle\left. n\right|_{\mathrm{ad}}(\varepsilon-\right.$ $\mathcal{H})|\Psi\rangle=0$ and find that the amplitude $\phi_{n}=$ $\psi_{\alpha_{n}} \gamma_{i} /\left(\varepsilon-\varepsilon_{i}\right)$ on the adsorbate is related to the amplitude of its host carbon site. The amplitudes $\phi_{n}$ hence can be eliminated, which results in the reduced Hamiltonian

$$
\tilde{\mathcal{H}}=-\gamma \sum_{\langle l, m\rangle} c_{l}^{\dagger} c_{m}+\sum_{n} V c_{\alpha_{n}}^{\dagger} c_{\alpha_{n}}, \quad V=\frac{\gamma_{i}^{2}}{\varepsilon-\varepsilon_{i}},
$$

where the energy-dependent effective potential $V$ corresponds to the self-energy which an adsorbate induces for electrons in graphene.

While the effective potential $V$ displays a distinct resonant energy dependence, the analysis of the resulting con- ductivity properties requires combining this with the specific energetics of the graphene sample, and, in particular, with the existence of the conical point at which the density of states drops to zero. We therefore now turn to the analysis for the Green's function $G=\left(\varepsilon-\mathcal{H}+i 0^{+}\right)^{-1}$ of the system.

For a single, well isolated adsorbate, the Green's function can be obtained exactly over the entire energy range by utilizing the $T$-matrix representation

$$
\mathcal{G}=G_{0}+G_{0} \mathcal{T} G_{0}, \quad \mathcal{T} \equiv\left(1-V c_{\alpha}^{\dagger} c_{\alpha} G_{0}\right)^{-1} V c_{\alpha}^{\dagger} c_{\alpha},
$$

where $G_{0}$ is the Green's function of the clean graphitic system and $\mathcal{T}$ characterizes the scattering strength [19]. Expanding $\mathcal{T}$ in powers of $V \mathcal{G}_{0}$, and utilizing the translational and crystalline symmetries of graphene (so that $\left\langle n\left|G_{0}\right| n\right\rangle \equiv g_{0}$ for all $n$ ), the resulting series for $G$ can be resummed yielding

$$
\mathcal{T}=t_{0}(\varepsilon) c_{\alpha}^{\dagger} c_{\alpha}, \quad t_{0}(\varepsilon)=\frac{\gamma_{i}^{2}}{\varepsilon-\varepsilon_{i}-\gamma_{i}^{2} g_{0}(\varepsilon)} .
$$

In the latter expression, $t_{0}(\varepsilon)$ describes resonant scattering of electrons in graphene from an adsorbate level renormalized by hybridization with states in the $\pi$ band. This hybridization always shifts the effective resonance level towards the Dirac point, which can be understood as a consequence of level repulsion which pushes additional states towards the region with the lowest density of states. The real part of $\gamma_{i}^{2} g_{0}(\epsilon)$ [with $\operatorname{Re} g_{0}(\varepsilon)=-\operatorname{Re} g_{0}(-\varepsilon) \approx$ $\left(A_{c} / 2 \pi \hbar^{2} v_{\mathrm{D}}^{2}\right) \varepsilon \ln (|\varepsilon| / \Delta)$, and $\Delta$ a high-energy cutoff] gives a formal description of this systematic shift, while the imaginary part [with $\operatorname{Im} g_{0}=-\pi \nu_{0}(\varepsilon)$, and $\nu_{0}(\varepsilon)$ the density of states per carbon atom] indicates a decrease of the resonance width near the Dirac point.

Ignoring (for the moment) the effects of phase-coherent multiple scattering off the adsorbates, the conductivity of a disordered sample with a finite adsorbate concentration $n_{i}$ can now be obtained in kinetic transport theory. Because of $C_{6}$ rotational symmetry of graphene, the conductivity is isotropic. Using the scattering rate obtained from (4),

$$
\tau_{\mathbf{k}}^{-1}=\left(\frac{2 \pi}{\hbar}\right) n_{i}\left|t_{0}\left(\varepsilon_{\mathbf{k}}\right)\right|^{2} \nu_{0}\left(\varepsilon_{\mathbf{k}}\right),
$$

where $\varepsilon_{\mathbf{k}}$ is the graphene dispersion relation, we find the conductivity of graphene in the presence of the adsorbates,

$$
\sigma=\left(\frac{g_{s} e^{2}}{h}\right) \frac{\hbar^{2}}{A_{c} n_{i}\left|t_{0}\left(\varepsilon_{\mathrm{F}}\right)\right|^{2}} \frac{\left\langle v_{\mathbf{k}}\right\rangle_{\varepsilon_{\mathrm{F}}}}{\left\langle v_{\mathbf{k}}^{-1}\right\rangle_{\varepsilon_{\mathrm{F}}}}
$$

where $\langle\cdots\rangle_{\varepsilon_{\mathrm{F}}}$ denotes integration along the Fermi line, $v_{\mathbf{k}} \equiv\left|\nabla_{\mathbf{k}} \varepsilon_{\mathbf{k}}\right| / \hbar$, and $g_{s}=2$ accounts for spin degeneracy.

Motivated by recent experimental findings for selectively adsorbed $\mathrm{H}^{+}$and $\mathrm{OH}^{-}$[3], we show in Fig. 2 the corresponding dependence of the conductivity in Eq. (6) on the Fermi energy (thick dashed curve). A key feature is the 


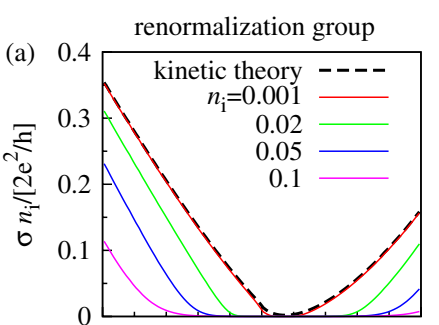

transport computations
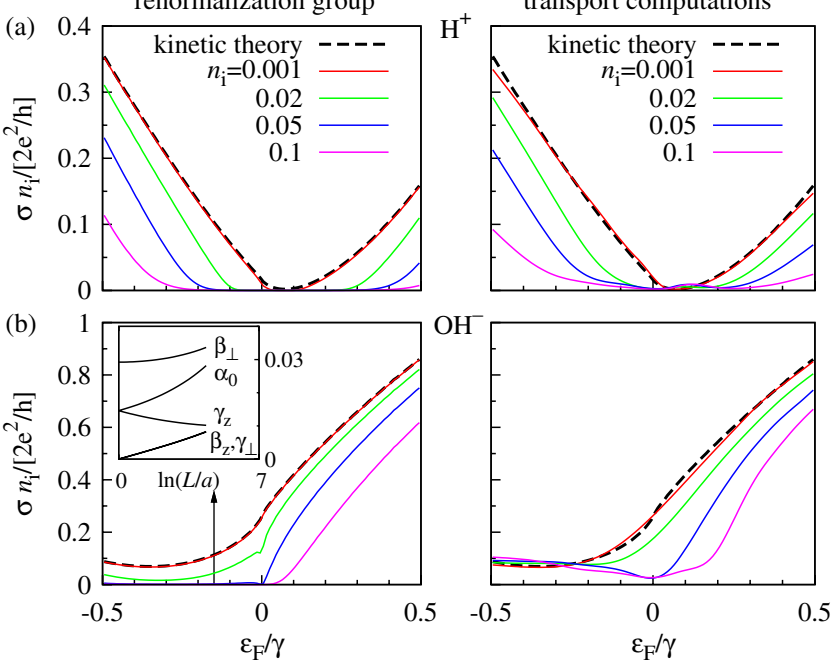

FIG. 2 (color online). Conductivity of graphene in the presence of adsorbates of variable concentration $n_{i}$. (a) $\mathrm{H}^{+}$, (b) $\mathrm{OH}^{-}$. The dashed thick curve is the prediction (6) of kinetic transport theory with energy-dependent $T$ matrix (4). The solid curves in the left panels show the expected quantum corrections based on the renormalization group analysis [Eqs. (10)]. In the right panels, the solid curves show the results of numerical transport computations. The inset in the left panel of (b) shows the RG flow (10) of the dimensionless scattering parameters for $\mathrm{OH}^{-}$at $\varepsilon=-0.15 \gamma$ and $n_{i}=0.01$.

marked asymmetry of the conductivity about the Dirac point caused by the energy dependence of the $T$ matrix (4). For $\mathrm{H}^{+}$, over a range of energies in the conduction band the conductivity is small, while it rises linearly as one moves into the valence band, or far into the conduction band. For $\mathrm{OH}^{-}$the role of the bands is reversed.

In actual devices, adsorbate scattering is supplemented by scattering off localized charges. These contribute to the scattering rate a term $\tau_{l}^{-1}=n_{l} \beta \gamma^{2} /(\hbar|\varepsilon|)$, where $n_{l}$ is the number of localized charges per carbon atom, and $\beta \simeq 1$ is a dimensionless numerical factor $[12,13]$. For energies in the linear part of the clean graphene dispersion relation, the resulting conductivity can be written in the form

$$
\sigma=\frac{2 \pi \sqrt{3}}{n_{l} \beta} \frac{g_{s} e^{2}}{h}\left[x\left|t_{0}\left(n_{e}\right) / \gamma\right|^{2}+n_{e}^{-1}\right]^{-1},
$$

where $x=(2 \pi / \beta)\left(n_{i} / n_{l}\right)$ characterizes the relative amount of the two types of disorder, and $n_{e}=$ $g_{s} \varepsilon_{\mathrm{F}}^{2} /\left(2 \sqrt{3} \pi \gamma^{2}\right)$ is the number of charge carriers per carbon atom. Figure 1 shows the charge-carrier dependence of the conductivity for various values of $x$. For $x=0$ the conductivity shows the symmetric linear charge-carrier dependence characteristic for charged impurity scattering. For an increasing adsorbate concentration, the conductivity develops the marked asymmetry discussed above, while the Coulomb scattering only dominates very close to the Dirac point, where it results in an additional dip.
Different types of disorder can also be discriminated via mesoscopic corrections to the conductivity, which originate in phase-coherent multiple scattering. In general, disorder in graphene can be characterized in terms of five dimensionless parameters $\Gamma=\left\{\alpha_{0}, \beta_{\perp}, \beta_{z}, \gamma_{\perp}, \gamma_{z}\right\}$, which classify the breaking of the symmetries of the honeycomb lattice [20]. Three of these parameters describe intravalley scattering preserving the $C_{6 v}$ symmetry $\left(\alpha_{0}\right)$, the $C_{3 v}$ symmetry $\left(\gamma_{z}\right)$, or no point symmetry $\left(\gamma_{\perp}\right)$, respectively. Analogously, intervalley scattering is described by the parameters $\beta_{z}$ and $\beta_{\perp}$. In terms of these parameters, the conductivity of graphene in kinetic theory takes the form

$$
\sigma=\frac{g_{s} e^{2}}{\hbar \pi^{2}}\left(\frac{\alpha_{0}}{2}+\beta_{\perp}+\gamma_{\perp}+\frac{3}{2} \beta_{z}+\frac{3}{2} \gamma_{z}\right)^{-1} .
$$

The scattering potential for adsorbates is such that

$$
\begin{aligned}
& \alpha_{0}=\gamma_{z}=\beta_{\perp} / 2=\frac{A_{c} n_{i}\left|t_{0}\left(\varepsilon_{\mathrm{F}}\right)\right|^{2}}{2 \pi\left\langle v_{\mathbf{k}}\right\rangle_{\varepsilon_{\mathrm{F}}} /\left\langle v_{\mathbf{k}}^{-1}\right\rangle_{\varepsilon_{\mathrm{F}}}}, \\
& \beta_{z}=\gamma_{\perp}=0 .
\end{aligned}
$$

This puts chemically functionalized graphene into the socalled orthogonal symmetry class, for which one expects a negative weak-localization magnetoresistance [20], as well as strong Anderson localization when the adsorbate concentration increases [14].

These corrections to kinetic theory can be studied systematically via a renormalization group analysis [21], which provides effective scattering parameters $\tilde{\Gamma}$ that replace the bare values in Eq. (8). For disorder representative of the symmetries in graphene, the flow equations were derived in Ref. [14]. An equivalent formulation in terms of the parameters $\Gamma$ given above can be found in Ref. [15], and takes the form

$$
\begin{aligned}
\dot{\alpha}_{0} & =2 \alpha_{0}\left(\alpha_{0}+\beta_{\perp}+\gamma_{\perp}+\beta_{z}+\gamma_{z}\right)+\beta_{\perp} \beta_{z}+2 \gamma_{\perp} \gamma_{z}, \\
\dot{\beta}_{\perp} & =4\left(\alpha_{0} \beta_{z}+\beta_{\perp} \gamma_{\perp}+\beta_{z} \gamma_{z}\right), \\
\dot{\beta}_{z} & =2\left(\alpha_{0} \beta_{\perp}-\beta_{z} \alpha_{0}+\beta_{\perp} \gamma_{z}+\beta_{z} \gamma_{z}\right), \\
\dot{\gamma}_{\perp} & =4 \alpha_{0} \gamma_{z}+\beta_{\perp}^{2}+\beta_{z}^{2}, \\
\dot{\gamma}_{z} & =2 \gamma_{z}\left(-\alpha_{0}-\beta_{\perp}+\beta_{z}+\gamma_{\perp}-\gamma_{z}\right)+2 \alpha_{0} \gamma_{\perp}+\beta_{\perp} \beta_{z}, \\
\dot{\varepsilon} & =\varepsilon\left(1+\alpha_{0}+\beta_{\perp}+\gamma_{\perp}+\beta_{z}+\gamma_{z}\right),
\end{aligned}
$$

where $\dot{X} \equiv d X / d \ln (L / a)$ and $L$ is a running length. This $\mathrm{RG}$ flow is integrated using the bare parameters $\Gamma$ and $\varepsilon=$ $\varepsilon_{\mathrm{F}}$ as initial conditions and terminated when $\varepsilon$ reaches a high-energy cutoff $\varepsilon_{c}$. We here implement this procedure for the energy-dependent initial conditions (9). A typical solution of the flow equations as a function of $\ln (L / a)$ is shown in the inset in Fig. 2(b) (left panel). The dominance of $\beta_{\perp}$ over $\gamma_{z}$ and $\gamma_{\perp}$ demonstrates that chemically functionalized graphene stays in the orthogonal symmetry class when the adsorbate concentration is increased. As shown 
by the curves in the left panels of Fig. 2, over the whole energy range and for both types of adsorbates $\left(\mathrm{H}^{+}\right.$and $\mathrm{OH}^{-}$) the renormalization leads to a suppression of the conductivity and drives the system towards insulating behavior.

In order to verify these expectations we carried out numerical transport computations, which are directly based on the model Hamiltonian (1). The conductivity $\sigma$ is obtained by finite-size scaling of the Landauer conductance $G=\sigma W / L$ of graphene ribbons with respect to width (in the range $50<W / \sqrt{3} a<100$ ) and length (in an adaptively chosen range that avoids the onset of Anderson localization [22]). For each geometry the conductance is computed in an efficient recursive Green's functions algorithm [23], and averaged over $10^{4}$ disorder realizations. The resulting dependence of the conductivity on the Fermi energy (right panels of Fig. 2) is in good agreement with the expectations based on the RG analysis (left panels of Fig. 2). In particular, the numerical results clearly confirm the suppression of the conductivity due to quantum corrections.

In summary, we have presented a theory of electronic transport for graphene in the presence of chemisorbed molecules. We find that each type of adsorbate results in a characteristic Fermi-energy dependence of the conductivity, which is asymmetric about the charge neutrality point and distinguishes $p$ - and $n$-type transport. In a range of energies, the conductivity is strongly suppressed, which could be used to increase the on-off ratio in graphenebased field-effect transistors. These effects are further enhanced by quantum corrections driving the system towards the localized state at higher adsorbate concentrations. In experiments, the adsorbate concentration can be increased by variable deposition times, or by driving adsorbates to the sample using top gates $[3,5,6]$, while the localization effects can be probed via magnetoresistivity experiments on graphene flakes at low temperatures [24].

We wish to thank Tim Echtermeyer for providing us with experimental data supporting the conclusions of this work, and gratefully acknowledge discussions with I. Aleiner, C. Lambert, A. Mirlin, and E. McCann. This work was supported by the European Commission, Marie Curie Excellence Grant MEXT-CT-2005-023778, and by the EPSRC.

[1] A. H. Castro Neto, F. Guinea, N.M.R. Peres, K. S. Novoselov, and A. K. Geim, arXiv:0709.1163 [Rev. Mod. Phys. (to be published)].

[2] F. Schedin, A. K. Geim, S. V. Morozov, D. Jiang, E. H. Hill, P. Blake, and K. S. Novoselov, Nature Mater. 6, 652 (2007).
[3] T. J. Echtermeyer, M.C. Lemme, M. Baus, B. N. Szafranek, A. K. Geim, and H. Kurz, IEEE Electron Device Lett. 29, 952 (2008).

[4] K. I. Bolotin, K. J. Sikes, Z. Jiang, M. Klima, G. Fudenberg, J. Hone, P. Kim, and H. L. Stormer, Solid State Commun. 146, 351 (2008).

[5] Z. Chen, Y. Lin, M. J. Rooks, and P. Avouris, Physica (Amsterdam) 40E, 228 (2007).

[6] M. C. Lemme, T. J. Echtermeyer, M. Baus, and H. Kurz, IEEE Electron Device Lett. 28, 282 (2007).

[7] J. H. Chen, C. Jang, M. S. Fuhrer, E. D. Williams, and M. Ishigami, Nature Phys. 4, 377 (2008).

[8] Conduction asymmetries can also arise for ionic bonding [7,9] and substitutional doping [10].

[9] D. S. Novikov, Appl. Phys. Lett. 91, 102102 (2007); M. Trushin and J. Schliemann, Europhys. Lett. 83, 17001 (2008).

[10] A. Lherbier et al., Phys. Rev. Lett. 101, 036808 (2008).

[11] R. Saito, G. Dresselhaus, and M. S. Dresselhaus, Physical Properties of Carbon Nanotubes (Imperial College Press, London, 1998).

[12] K. Nomura and A. H. MacDonald, Phys. Rev. Lett. 98, 076602 (2007); V. V. Cheianov and V. I. Fal'ko, Phys. Rev. Lett. 97, 226801 (2006).

[13] E. H. Hwang, S. Adam, and S. Das Sarma, Phys. Rev. Lett. 98, 186806 (2007).

[14] I. L. Aleiner and K. B. Efetov, Phys. Rev. Lett. 97, 236801 (2006); M.S. Foster and I. L. Aleiner, Phys. Rev. B 77, 195413 (2008).

[15] P. M. Ostrovsky, I. V. Gornyi, and A. D. Mirlin, Phys. Rev. B 74, 235443 (2006).

[16] J. M. Soler, E. Artacho, J. D. Gale, A. García, J. Junquera, P. Ordejón, and D. Sánchez-Portal, J. Phys. Condens. Matter 14, 2745 (2002).

[17] L. Jeloaica and V. Sidis, Chem. Phys. Lett. 300, 157 (1999).

[18] We also ignore effects of adsorbate-induced magnetism such as studied, e.g., in D.W. Boukhvalov, M.I. Katsnelson, and A.I. Lichtenstein, Phys. Rev. B 77, 035427 (2008); B. Uchoa, V. N. Kotov, N. M. R. Peres, and A.H. Castro Neto, Phys. Rev. Lett. 101, 026805 (2008).

[19] For static impurities see, e.g., Yu. V. Skrypnyk and V. M. Loktev, Phys. Rev. B 73, 241402(R) (2006); D. M. Basko, Phys. Rev. B 78, 115432 (2008).

[20] E. McCann, K. Kechedzhi, Vladimir I. Fal'ko, H. Suzuura, T. Ando, and B. L. Altshuler, Phys. Rev. Lett. 97, 146805 (2006).

[21] A. W. W. Ludwig, M. P. A. Fisher, R. Shankar, and G. Grinstein, Phys. Rev. B 50, 7526 (1994).

[22] J.P. Robinson and H. Schomerus (to be published).

[23] J. P. Robinson and H. Schomerus, Phys. Rev. B 76, 115430 (2007).

[24] F. V. Tikhonenko, D. W. Horsell, R. V. Gorbachev, and A. K. Savchenko, Phys. Rev. Lett. 100, 056802 (2008).

[25] The limit $\left|n_{e}\right|=0.005$ of the range plotted in Fig. 1 corresponds to a charge-carrier density $\left|n_{e}\right| / A_{c}=1.9 \times$ $10^{13} \mathrm{~cm}^{-2}$ and a Fermi energy $\left|\varepsilon_{\mathrm{F}}\right|=0.165 \gamma$. 